Preprint JINR E2-93-225

\title{
Antibrackets and Supersymmetric Mechanics
}

\author{
Armen Nersessian 巴 \\ Laboratory of Theoretical Physics, JINR \\ Dubna, Head Post Office, P.O.Box 79, 101000 Moscow, Russia
}

\begin{abstract}
Using odd symplectic structure constructed over tangent bundle of the symplectic manifold, we construct the simple supergeneralization of an arbitrary Hamiltonian mechanics on it. In the case, if the initial mechanics defines Killing vector of some Riemannian metric, corresponding supersymmetric mechanics can be reformulated in the terms of even symplectic structure on the supermanifold.
\end{abstract}

\footnotetext{
${ }^{1}$ E-MAIL:NERSESS@THEOR.JINRC.DUBNA.SU
} 


\section{Introduction}

It is well-known that on supermanifolds $\mathcal{M}$ the Poisson brackets of two types can be defined - even and odd ones, in correspondence with their Grassmannian grading ${ }^{1}$. That is defined by the expression

$$
\{f, g\}_{\kappa}=\frac{\partial_{r} f}{\partial z^{A}} \Omega_{\kappa}^{A B}(z) \frac{\partial_{l} g}{\partial z^{B}}
$$

which satisfies the conditions

$$
\begin{aligned}
& p\left(\{f, g\}_{\kappa}\right)=p(f)+p(g)+1 \quad \text { (grading condition), } \\
& \{f, g\}_{\kappa}=-(-1)^{(p(f)+\kappa)(p(g)+\kappa)}\{g, f\}_{\kappa} \quad \text { ("antisymmetrisity"), } \\
& (-1)^{(p(f)+\kappa)(p(h)+\kappa)}\left\{f,\{g, h\}_{1}\right\}_{1}+\text { cycl.perm.(f, g, h) }=0 \quad \text { (Jacobi id.), }
\end{aligned}
$$

where $z^{A}$ are the local coordinates on $\mathcal{M}, \frac{\partial_{r}}{\partial z^{A}}$ and $\frac{\partial^{l}}{\partial z^{A}}$ denote correspondingly the right and the left derivatives, $\kappa=0,1$ denote correspondingly the even and the odd Poisson brackets.

Obviously, the even Poisson brackets can be nondegenerate only if $\operatorname{dim} \mathcal{M}=(2 N . M)$, and the odd one if $\operatorname{dim} \mathcal{M}=(N . N)$.

With nondegenerate Poisson bracket one can associate the symplectic structure

$$
\Omega_{\kappa}=d z^{A} \Omega_{(\kappa) A B} d z^{B}
$$

where $\Omega_{(\kappa) A B} \Omega_{\kappa}^{B C}=\delta_{A}^{C}$.

Locally nondegenerate even and odd Poisson brackets can be reduced correspondingly to the forms ${ }^{2,3}$ :

$$
\{f, g\}_{0}^{\text {can }}=\sum_{i=1}^{N}\left(\frac{\partial f}{\partial x^{i}} \frac{\partial g}{\partial x^{i+N}}-\frac{\partial f}{\partial x^{i+N}} \frac{\partial g}{\partial x^{i}}\right)+\sum_{\alpha=1}^{M} \epsilon_{\alpha} \frac{\partial_{r} f}{\partial \theta_{\alpha}} \frac{\partial_{l} g}{\partial \theta_{\alpha}}, \quad \epsilon_{\alpha}= \pm 1
$$

and

$$
\{f, g\}_{1}^{\mathrm{can}}=\sum_{i=1}^{N}\left(\frac{\partial_{r} f}{\partial x^{i}} \frac{\partial_{l} g}{\partial \theta_{i}}+\frac{\partial_{r} f}{\partial \theta_{i}} \frac{\partial_{l} g}{\partial x^{i}}\right) .
$$

The even Poisson brackets are the straightforward generalization of the ordinary Poisson brackets on the manifolds. They are widely used in physics for the description of the Hamiltonian systems. After quantization such systems described the theories containing both fermionic (real and ghosts) and bosonic degrees of freedom.

The odd Poisson brackets were introduced in field theory by Batalin and Vilkovisky ${ }^{4}$ (they called them antibrackets, and following thats, we will use the same name) for the formulation of the covariant (Lagrangian) quantization formalism for the field theories with arbitrary constraints (BV-formalism). BV-formalism and its generalizations ${ }^{5}$ are most general methods of the quantization of the gauge theories. Recently interest has been aroused in its investigation. It was stimulated by the papers ${ }^{6}$, which started developing the background-independent string field theory on the basis of BV-formalism . 
However, the antibrackets can be also used in the direct way - for the formulation of the Hamiltonian mechanics with antibrackets ( anti-Hamiltonian mechanics) :

$$
\frac{d z^{A}}{d t}=\left\{z^{A}, Q\right\}_{1}, \quad p(Q)=1
$$

Leites ${ }^{2}$ was the first to point out the possibility of the formulation of such Hamiltonian mechanics.

However, there were a number of obstacles in the quantization of the anti- Hamiltonian mechanics and in the physical interpretation of its grassmannian degrees of freedom: the violated spin-statistics coupling, the necessity of introduction of the odd Planck constant etc.

Possibilities to get over such difficulties were pointed out by D. V. Volkov et al. ${ }^{7,8}$. Particularly, in ${ }^{8}$ it was shown that one-dimensional Witten's supersymmetric mechanics can be described using antibrackets, and the role of Hamiltonian in this case plays one of its supercharges.

Later the Hamiltonian systems, provided with both even and odd Poisson brackets were studied in more detail in ${ }^{9}$.

Nevertheless, the absence of the physical examples, where introduction of the structure of anti-Hamiltonian mechanics was necessary, or at least successful, gave them the status of non-interests systems.

Resently in ${ }^{10,11}$ the new method of the exact evaluation of the Hamiltonian path integrals was developed. It is based on the generalization of the Duistermaat- Heckman localization formula ${ }^{12}$. Using this generalization, one can localize the path integral to the finite-dimensional integral over classical phase space. This forms the basis for the conceptually new approach to the invariant description of supersymmetric theories.

$\mathrm{In}^{13}$ it was shown that it is convenient to use for the description of this method the odd symplectic structure, constructed on the supermanifold associated with the tangent bundle of symplectic manifold, and the corresponding Hamiltonian dynamics (antiHamiltonian dinamics).

Parallelly, this gives the supersymmetrization method for a wide class of the Hamiltonian systems (namely, for the Hamiltonian systems which define Killing vectors of the some Riemannian metrics on the phase space), for which there exists a way to go round the noted difficulties connected with the quantization.

In the present paper we shall study such Hamiltonian systems and their supersymmetrization method more closely.

The paper is organized in the following way:

In Section 2 we shall construct the odd symplectic structure and the corresponding antibrackets on the supermanifold, associated with the tangent bundle of the initial symplectic manifold. Then we shall define the natural map of the mechanics on the initial manifold in the supersymmetric anti-Hamiltonian mechanics, and interpret this supersymmetry in terms of the basic manifold.

In Section 3 we shall show that if the initial mechanics defines the Killing vector of some Riemannian metrics on the phase space, then the corresponding supersymmetric anti-Hamiltonian mechanics can be reformulated with the even Poisson brackets. 


\section{Anti-Hamiltonian Systems and Supersymmetry}

Let $M$ be the manifold with the symplectic structure

$$
\omega=\frac{1}{2} \omega_{i j} d x^{i} \wedge d x^{j}
$$

and

$$
\{f(x), g(x)\}=\frac{\partial f}{\partial x^{i}} \omega^{i j} \frac{\partial g}{\partial x^{j}}
$$

is the corresponding nondegenerate Poisson bracket on it.

The Hamiltonian $H(x)$ defines on it the Hamiltonian mechanics with the equations of motion:

$$
\frac{d x^{i}}{d t}=\left\{x^{i}, H(x)\right\} \equiv \xi^{i} .
$$

It is known that any supermanifold can be associated with some vector bundle ${ }^{1}$. On the supermanifold which is associated with the cotangent bundle of any manifold, one can construct the odd symplectic structure ${ }^{2}$ ( corresponding Poisson brackets known in mathematics as Schowten brackets). Indeed, let $\mathcal{M}$ be the supermanifold, associated with $M$. Then on every map on $\mathcal{M}$ one can choose the local coordinates $\left(x^{i}, \theta_{i}\right)\left(p\left(\theta_{i}\right)=\right.$ $p\left(x^{i}\right)+1=1$ ), which are transformed from map to map in accordance with

$$
x^{i} \rightarrow \tilde{x}^{i}=\tilde{x}^{i}(x), \quad \theta_{i} \rightarrow \tilde{\theta}_{i}=\sum_{i=1}^{N} \frac{\partial x^{j}}{\partial \tilde{x}^{i}} \theta_{j},
$$

i.e. $\theta_{i}$ corresponds to $\frac{\partial}{\partial x^{i}}$.

Then, obviously, using these coordinates, one can globally define on $\mathcal{M}$ the antibrackets (1.6). Let us map the functions on $M$ onto the odd ones on $\mathcal{M}$ :

$$
f(x) \rightarrow Q_{f}(z)=\{f(x), F(x, \theta)\}_{1},
$$

where

$$
F(z)=\frac{1}{2} \theta_{i} \omega^{i j} \theta_{j}
$$

corresponds to the Poisson bracket (2.2) on $M$.

It is easy to see that

$$
\{f(x), g(x)\}=\left\{f(x), Q_{g}(x, \theta)\right\}_{1} \quad \text { for } \quad \text { any } \quad f(x), g(x) .
$$

Then, the map (2.5) puts the Hamiltonian mechanics $(H, \omega, M)$ into the anti-Hamiltonian mechanics $\left(Q, \Omega_{1}, \mathcal{M}\right)$, where

$$
Q \equiv Q_{H}=\{H, F\}_{1}
$$

This mechanics is supersymmetric. Indeed, it is easy to see that the functions $H, F, Q$ form the simplest superalgebra:

$$
\begin{array}{ll}
Q=\{H, F\}_{1}, & \{H, H\}_{1}=0, \quad\{F, F\}_{1}=0, \\
\{Q, Q\}_{1}=0, & \{H, Q\}_{1}=\{F, Q\}_{1}=0,
\end{array}
$$


or, equivalently,

$$
\begin{aligned}
& \{H \pm F, H \pm F\}_{1}= \pm 2 Q, \\
& \{H+F, H-F\}_{1}=\{H \pm F, Q\}_{1}=\{Q, Q\}_{1}=0 .
\end{aligned}
$$

The last equation in (2.9) corresponds to the Jacobi identity for (2.2).

For the interpretation of this superalgebra note that in (2.5) $Q_{f}$ is transformed as $d f$. Correspondingly,

$$
\theta^{i}=\left\{x^{i}, F\right\}_{1}=\omega^{i j} \theta_{j}
$$

can be interpreted as the basic 1-forms $d x^{i}$.

Then, one can pass from the desctiption in terms of the coordinates $\left(x^{i}, \theta_{i}\right)$ to that in terms of the coordinates $\left(x^{i}, \theta^{i}\right)$.

Obviously, any function $f\left(x^{i}, \theta^{i}\right)$ can be interpreted in terms of differential forms on $M$.

In terms of $\left(x^{i}, \theta^{i}\right)$ antibrackets (1.6) on $\mathcal{M}$ take the form:

$$
\left\{x^{i}, x^{j}\right\}_{1}=0, \quad\left\{x^{i}, \theta^{j}\right\}_{1}=\omega^{i j}, \quad\left\{\theta^{i}, \theta^{j}\right\}_{1}=\frac{\partial \omega^{i j}}{\partial x^{k}} \theta^{k}
$$

where $\omega^{i j}$ is the matrix of the even Poisson bracket (2.2) on $M$.

The corresponding odd symplectic structure in coordinates $z^{A}=\left(x^{i}, \theta^{i}\right)$ takes the form

$$
\Omega_{1}=\frac{1}{2} \omega_{i j} d x^{i} \wedge d \theta^{j}+\frac{1}{2} \omega_{i j, k} \theta^{k} d x^{i} \wedge d x^{j}
$$

The equations of motion of the anti-Hamiltonian mechanics $\left(Q, \Omega_{1}, \mathcal{M}\right)$ are :

$$
\frac{d x^{i}}{d t}=\left\{x^{i}, Q\right\}_{1}=\xi^{i} \quad \frac{d \theta^{i}}{d t}=\left\{\theta^{i}, Q\right\}_{1}=\frac{\partial \xi^{i}}{\partial x^{j}} \theta^{j} .
$$

The following correspondence is obvious

$$
\begin{aligned}
& \{H, \quad\}_{1}=\xi^{i} \frac{\partial}{\partial \theta^{i}} \rightarrow \imath_{H}-\text { operator of inner product on } \xi^{i} \\
& \{F, \quad\}_{1}=\theta^{i} \frac{\partial}{\partial x^{i}} \rightarrow d \text {-operator of exterior differentiation; } \\
& \{Q, \quad\}_{1}=\xi^{i} \frac{\partial}{\partial x^{i}}+\xi_{, k}^{i} \theta^{k} \frac{\partial}{\partial \theta^{i}} \rightarrow \mathcal{L}_{H}-\text { Lie derivative along } \xi^{i}
\end{aligned}
$$

Then, using the Jacobi identity (1.3), we show

$$
\{H, F\}_{1}=Q \rightarrow d \imath_{H}+\imath_{H} d=\mathcal{L}_{H}-\text { homotopy formula }
$$

As we see, the supercharge $H+F$ which defines the supersymmetry transformation , corresponds to the operator of the equivariant differentiation $d_{H}=d+\imath_{H}$.

Therefore the presented anti-Hamiltonian mechanics is the natural canditate for the description of equivariant localization ${ }^{13}$.

However, and we noted that in the Introduction, there are many obstacles in the quantization and interpretation of the anti-Hamiltonian mechanics. In the next Section we shall show that some additional assumption about the anti-Hamiltonian mechanics (2.14) allows reformulate it with the even Poisson brackets and to go round this obstacles. 


\section{Reformulation with Even Poisson Brackets}

In the previous Section we saw, that defining the map (2.5), we go from the arbitrary Hamiltonian mechanics (2.3) to the supersymmetric one (2.14).

In this Section we shall consider the special case of the mechanics (2.3), when it defines the Killing vector for some Riemannian mertic on the phase space.

In this case the corresponding supersymmetrization (2.14) can be reformulated in terms of the even symplectic structure on the phase superspace.

Let us assume that the manifold $M$ is provided with both the symplectic structure $\omega_{\alpha}$ and the Riemannian one $g_{i j}$.

Let the local 1-form $A_{\alpha}=A_{(\alpha) i} d x^{i}$ define on $M$ this symplectic structure:

$$
d A_{\alpha}=\omega_{\alpha}
$$

Then consider on $\mathcal{M}$ the following local 1-form:

$$
\mathcal{A}_{\alpha}=A_{(\alpha) i} d x^{i}+\theta^{i} g_{i j} D \theta^{j},
$$

where $D \theta^{i}=d \theta^{i}+\Gamma_{k l}^{i} \theta^{k} d x^{l}$ and $\Gamma_{k l}^{i}$ - the Cristoffel symbols for metrics $g_{i j}$ on $M$.

The exterior differential of this 1-form (globally) define on $\mathcal{M}$ the even symplectic structures:

$$
\Omega_{\alpha}=d \mathcal{A}_{\alpha}=\frac{1}{2}\left(\omega_{(\alpha) i j}+R_{i j k l} \theta^{k} \theta^{l}\right) d x^{i} \wedge d x^{j}+g_{i j} D \theta^{i} \wedge D \theta^{j},
$$

where $R_{i j k l}$ - the curvature tensor on $M$.

The Poisson brackets, which correspond to this structure, are :

$$
\{f(z), g(z)\}_{\alpha}=\nabla_{i} f(z)\left(\omega_{(\alpha) i j}+R_{i j k l} \theta^{k} \theta^{l}\right)^{-1} \nabla_{j} g(z)+\frac{\partial_{r} f(z)}{\partial \theta^{i}} g^{i j} \frac{\partial_{l} g(z)}{\partial \theta^{j}},
$$

where $g^{i k} g_{k j}=\delta_{j}^{i}$,

$$
\nabla_{i}=\frac{\partial}{\partial x^{i}}-\Gamma_{i j}^{k} \theta^{j} \frac{\partial_{l}}{\partial \theta^{k}}
$$

Now let us assume that the Riemannian metric $g_{i j}$ on $M$ Lie-derived with $\xi^{i}(2.3)$ :

$$
\mathcal{L}_{H} g=0 \Leftrightarrow \xi_{, i}^{k} g_{k j}+g_{i k} \xi_{, j}^{k}+g_{i j, k} \xi^{k}=0
$$

Then the odd function

$$
\tilde{Q}=\xi^{i} g_{i j} \theta^{j}
$$

on $\mathcal{M}$ is the motion integral of the anti-Hamiltonian mechanics:

$$
\mathcal{L}_{H} g=0 \rightarrow\{Q, \tilde{Q}\}_{1}=0
$$

The functions $F$ and $H$ commute with $\tilde{Q}$ in the following way:

$$
\{F, \tilde{Q}\}_{1}=-F_{2}, \quad\{H, \tilde{Q}\}_{1}=H_{2},
$$


where

$$
H_{2}=\xi^{i} g_{i j} \xi^{j}, \quad F_{2}=\frac{1}{2} \theta^{i} \omega_{(2) i j} \theta^{j}, \quad \omega_{(2) i j}=\frac{\partial\left(g_{i k} \xi_{H}^{k}\right)}{\partial x^{j}}-\frac{\partial\left(g_{j k} \xi_{H}^{k}\right)}{\partial x^{i}}
$$

Let us assume that $\operatorname{det} \omega_{(2) i j} \neq 0$.

Then, the mechanics $\left(H_{2}, \omega_{(2) i j} d x^{i} \wedge d x^{j}, M\right)$ and $(H, \omega, M)$ define the bi-Hamiltonian structure on $M$ (it was first pointed out first in $\left.{ }^{12}\right)$ :

$$
\xi^{i}=\omega^{i j} \frac{\partial H}{\partial x^{j}}=\omega_{(2)}^{i j} \frac{\partial H_{2}}{\partial x^{j}} .
$$

It is easy to see that $\left(\mathcal{H}_{\alpha}=H_{\alpha}+F_{2}, \Omega_{\alpha}, \mathcal{M}\right)$ and $\left(Q, \Omega_{1}, \mathcal{M}\right)$ define the same Hamiltonian dynamics (2.14) on $\mathcal{M}$.

There $\alpha=0,2$ and $\Omega_{\alpha}$ is defined by the (3.2), $\omega_{0} \equiv \omega, H_{0} \equiv H$.

In other words, we provide the supersymmetric anti-Hamiltonian mechanics (2.14) with the even Hamiltonian structure in the case, that the initial mechanics $(H, \omega, M)$ define the Killing vector for the some Riemannian metric on the $M$.

Using the description with it we can go round the obstacles connected with the difficultes in the quantization and interpretation of anti-Hamiltonian systems.

However, on the level of classical description the use of antibrackets make its more simple and transparent.

It is obvious that this dynamics has at least two supercharges.

We have got the simple supersymmetrization method for the Hamiltonian systems, which is defined on the symplectic manifolds, provided with the Riemannian structures, Lie-derived with it.

This class includes the integrable systems on the orbits of the coadjoint representation of semisimple Lie groups and therefore, really all integrable systems of classical mechanics.

Example (1D supersymmetric Witten mechanics) of dynamics with even and odd Hamiltonian structures was considered at first in ${ }^{8}\left(\right.$ see also $\left.^{9}\right)$.

\section{References}

1. F. A. Berezin - Introduction to Superanalysis., D. Reidel, Dordrecht, 1986

T. Voronov - Geometric Integration Theory on Supermanifolds. Sov. Sci. Rev. C, Math.Phys., v.9, 1992

2. D. A. Leites - Dokl. Akad. Nauk SSSR, 236 (1977), 804

3. V. N. Shander - Dokl. Akad. Nauk. Bulgaria , (1983)

4. I. A. Batalin , G. A. Vilkovisky - Phys.Lett., 102B (1981), 27; Nucl.Phys., B234 (1984), 106

5. I. A. Batalin, P. M. Lavrov, I. V. Tyutin -J. Math. Phys., 31 (1990) 1487; ibid. 32 (1991), 532; ibid. 32 (1991), 2513

I. A. Batalin, I. V. Tyutin - Preprint FIAN/TD/18-92, Int. J. Mod. Phys. A , (1993) 
6. E. Witten-Mod. Phys. Lett. A, 5 (1990), 487; Preprint IASSNS-HEP- 92/53 ; Preprint IASSNS-HEP- 92/63

7. D. V. Volkov - JETP Lett., 38 (1983), 508

D. V. Volkov , V. A. Soroka , V. I. Tkach - Sov. J. Nucl. Phys., 46 (1987) , 110

8. D. V. Volkov, V. A. Soroka, A. I. Pashnev, V. I.Tkach - JETP Lett., 44 (1986), 55

9. O. M. Khudaverdian, A. P. Nersessian - Preprint YERPHI-1031(81)-1987; J. Math. Phys., 32 (1991), 1938 ; Preprint JINR E2-92-411, J. Math. Phys.(to appear)

O. M. Khudaverdian - J. Math. Phys., 32 (1991), 1934 ;

A. P. Nersessian - Theor. Math. Phys.,96 (1993), No. 1 (in press)

10. M. Blau, E. Keski- Vakkuri , A. J. Niemi - Phys.Lett., 246B (1990), 92;

A. Hietaki, A. Yu. Morozov, A. J. Niemi, K. Palo - Phys. Lett. B263 (1991), 417 A. Yu. Morozov, A. J. Niemi, K. Palo - Phys. Lett B271 (1991), 365; Nucl. Phys. B377 (1992), 295

A. J. Niemi, O. Tirkkonen - Phys.Lett., 293B (1992), 339;

E. Witten - Preprint IASSNS-HEP-92/15

11. A. J. Niemi, O. Tirkkonen - Preprint UU-ITP 3/93

12. J. J. Duistermaat, G. J. Heckman - Inv. Math. 69 (1982), 259; ibid.72 (1983), 153 M. F. Atiah , R. Bott - Topology, 23, No. 1 (1984), 1

13. A.P. Nersessian - JETP Lett., 58 (1993), No. 1 (in press); 